\title{
ASSESSING HEALTHCARE PROVIDERS' PERFORMANCE WITH AND WITHOUT RISK ADJUSTMENT
}

\author{
DANIEL MAURICIO MORALES-SILVA ${ }^{\circledR}$
}

(Received 23 March 2020; first published online 6 May 2020)

2010 Mathematics subject classification: primary 62-07.

Keywords and phrases: data analysis, health informatics.

This thesis focuses on how healthcare data can be used to draw comparisons between healthcare providers (surgeons or hospitals). Depending on the type of access to datasets, these comparisons can be done with or without risk adjustment. Risk adjustment refers to the use of patient-level information to explain variation in healthcare spending, resource utilisation and health outcomes.

For unadjusted comparisons, we highlight the diagnostic potential that radar plots offer for reporting on outcome indicators. These outcome indicators were obtained from hospital admissions of patients undergoing certain surgical procedures. We address two drawbacks of radar plots: the presence of missing information and the order of indicators. By introducing a consolidated view at provider level, we define an uncomplicated ranking of providers which can be used to identify potential low and high performers.

For risk-adjusted comparisons, we introduce a novel and robust methodology that enables comparisons of healthcare providers across multiple hierarchies, namely, surgeons, teams, departments and hospitals, using a consistent approach. Our methodology puts the patient at the centre of the analysis, and thus can be used for personalised predictions (for example, expected length of stay, costs and probability of being transferred to the intensive care unit).

Our findings suggest that the observed variation in selected outcome indicators, such as length of stay and charges of healthcare providers, cannot be explained by patient characteristics alone. Importantly, we have also observed that the perceived performance of providers on selected outcome indicators can change substantially following risk adjustment.

Thesis submitted to Federation University in September 2018; degree approved on 16 January 2019; supervisor Guillermo Pineda-Villavicencio.

(C) 2020 Australian Mathematical Publishing Association Inc. 
Healthcare is unique in that clinical expertise is essential in guiding decisionmaking and in informing all statistical models that seek to describe patient outcomes. For future iterations of our models, we will seek greater clinical input.

Some of the research in this thesis has been published in [1].

\section{Reference}

[1] D. M. Morales-Silva, C. S. McPherson and G. Pineda-Villavicencio, 'Using radar plots for performance benchmarking at patient and hospital levels using an Australian orthopaedics dataset', Health Inform. J., to appear. Published online (20 January 2020).

DANIEL MAURICIO MORALES-SILVA, School of Science, Engineering and Information Technology, Federation University, Ballarat, Victoria 3353, Australia

e-mail: d.moralessilva@federation.edu.au 